\title{
Chemiluminescence and superoxide production in Acanthamoeba castellanii: free radicals generated during oxidative stress
}

\author{
Bernard Davies and Steven W. Edwards* \\ Department of Biochemistry, University of Liverpool, PO Box 147, Liverpool L69 3BX, UK
}

(Received 15 November 1990; revised 5 February 1991; accepted 11 February 1991)

\begin{abstract}
The amoeba Acanthamoeba castellanii generated both luminol- and lucigenin-enhanced chemiluminescence upon addition of the respiratory inhibitor sodium cyanide, but not upon the addition of sodium azide. Photon emission in the presence of lucigenin was three- to fourfold greater than that measured in the presence of luminol, but both forms of chemiluminescence were strictly dependent upon the presence of $\mathrm{O}_{2}$, indicating the requirement for oxidative reactions in these processes. Lucigenin-chemiluminescence measured during the phagocytosis of latex beads under identical conditions was, however, barely detectable above background levels. Cyanide similarly induced the formation of $\mathrm{O}_{2}^{-}$, as indicated by the stimulation of superoxide-dismutase-inhibitable cytochrome $c$ reduction, and the rate of production was similar to that previously observed during phagocytosis of latex particles or yeasts by these cells. Thus, in view of the similar rates of $\mathrm{O}_{2}^{-}$production during cyanide exposure or phagocytosis, but disparate rates of lucigenin-chemiluminescence, these two treatments must activate different molecular processes leading to reactive oxidant production. The rates of cyanide-stimulated lucigeninchemiluminescence were directly proportional to the $\mathrm{O}_{2}$ tensions in the medium from 0 to $300 \mu \mathrm{M}$, indicating that the rates of free-radical-generating reactions were directly related to the oxygen tensions in the environment. The superoxide dismutase inhibitor diethyldithiocarbamate similarly stimulated lucigenin-chemiluminescence, with photon emission again being dependent upon $\mathrm{O}_{2}$ tensions in the range 0-320 $\mu \mathrm{M}$. A mechanism by which cells may limit $\mathrm{O}_{2}^{-}$-generating reactions, and so reduce damaging free radical reactions, was observed when anaerobic suspensions were re-aerated. These data indicate that oxidative stress and phagocytosis provide two intracellular sources of free-radical generating reactions in these cells.
\end{abstract}

\section{Introduction}

Acanthamoeba castellanii is a phagocytic soil amoeba which possesses a complex array of terminal oxidases whose relative activities and contributions to whole-cell respiration show considerable variations during exponential growth. These oxidases include a mitochondrial (cyanide-sensitive) cytochrome oxidase, an alternative salicylhydroxamic-acid-sensitive (cyanide-insensitive) mitochondrial oxidase and a low-affinity cyanidesensitive (azide-insensitive) component (Edwards \& Lloyd, 1977, 1978; Lloyd et al., 1979a). Additionally, these cells possess a cyanide-insensitive $\mathrm{O}_{2}^{-}$-generating oxidase (resembling the NADPH oxidase of neutrophils and macrophages; Curnutte, 1988), which becomes activated during phagocytosis of heat-killed yeast and latex particles (Davies et al., 1991). This latter oxidase is responsible for the lucigenin-dependent chemiluminescence which is produced during this process. A $b$-type cytochrome (Segal \& Jones, 1978) becomes associated with phagolysosomal membranes during engulfment of latex particles (Davies et al., 1991) and hence the $\mathrm{O}_{2}^{-}-$ generating oxidases of $A$. castellanii and of 'professional phagocytes' may be structurally related.

The respiratory burst of 'professional phagocytes' may be monitored by the sensitive technique of chemiluminescence, which can be amplified using probes such as luminol (Allen \& Loose, 1976) and lucigenin (Allen, 1981). The precise molecular processes that result in photon emission during oxidation of these probes are unknown, but the former measures peroxidase $/ \mathrm{H}_{2} \mathrm{O}_{2}-$ dependent reactions whereas the latter appears to be dependent upon $\mathrm{O}_{2}^{-}$and/or $\mathrm{H}_{2} \mathrm{O}_{2}$ formation (Edwards, 1987). The $\mathrm{O}_{2}^{-}$-generating oxidase of $\boldsymbol{A}$. castellanii, unlike that of neutrophils and macrophages, produces little, if any, luminol-chemiluminescence during phagocytosis (because the cells are devoid of peroxidase), but lucigenin-chemiluminescence is readily detected (Davies et al., 1991).

Previous work has shown that when cultures of $A$. 
castellanii are exposed to the respiratory inhibitor sodium cyanide, they generate low levels of chemiluminescence (Lloyd et al., 1979b). It was proposed that this $\mathrm{O}_{2}$ sensitive photon emission was due to inhibition by cyanide of superoxide dismutase, the enzyme responsible for regulating the intracellular levels of $\mathrm{O}_{2}^{-}$which is inevitably generated during oxidative metabolism (Halliwell \& Gutteridge, 1985). Inhibition of superoxide dismutase by cyanide thus results in an increase in the steady-state level of $\mathrm{O}_{2}^{-}$and this oxidant (or an oxidant derived from it, such as a product of lipid peroxidation) is responsible for photoemission.

The aim of this work was to identify the mechanisms by which $\mathrm{O}_{2}^{-}$may be generated in these cells and to ascertain if cyanide-stimulated chemiluminescence was mediated by molecular processes which were similar to, or distinct from, those involved in the respiratory burst.

\section{Methods}

Growth and maintenance of the organism. A. castellanii was grown and maintained with shaking at $30^{\circ} \mathrm{C}$ exactly as described previously (Edwards \& Lloyd, 1977). The growth medium comprised $(\%, w / v)$ : proteose peptone, 0.75 ; yeast extract, 0.75 ; glucose, 1.5 . Organisms were counted (after a suitable dilution with $0.9 \% \mathrm{NaCl}$ ) using a Fuchs Rosenthal haemocytometer slide.

Analytical methods. Superoxide anion $\left(\mathrm{O}_{2}^{-}\right)$production by cell suspensions in PBS (phosphate-buffered saline: $0.9 \% \mathrm{NaCl} ; 10 \mathrm{mM}$ potassium phosphate buffer; $\mathrm{pH} 7.4$ ) was measured by the method of Babior et al. (1973) using a Perkin-Elmer Lambda 5 spectrophotometer: suspensions $(1 \mathrm{ml})$ contained $1 \times 10^{6}$ cells, $75 \mu \mathrm{M}$-cytochrome $c$, and superoxide dismutase, as indicated. Chemiluminescence measurements were performed in $10 \mathrm{ml}$ suspensions containing either $10 \mu \mathrm{M}^{-}$ luminol or $50 \mu \mathrm{M}$-lucigenin in a specially constructed 'open' system in which $\mathrm{O}_{2}$ measurements could be made simultaneously with chemiluminescence measurements (Edwards et al., 1987). In this system the $\mathrm{O}_{2}$ tensions in the gas phase (and after equilibration, those in the liquid phase) were regulated using a digital gas mixer.

\section{Results}

\section{Effect of cyanide and azide on chemiluminescence}

Suspensions of $A$. castellanii were grown to the midexponential phase of growth $\left(1-2 \times 10^{6}\right.$ cells ml $\left.^{-1}\right)$ and suspended in PBS. Ten millilitres of suspension were placed into the 'open' $\mathrm{O}_{2} /$ chemiluminescence system (maintained at $30^{\circ} \mathrm{C}$ ) and lucigenin was added to $50 \mu \mathrm{M}$. Under a gas phase of air $\left(235 \mu \mathrm{M}-\mathrm{O}_{2}\right.$ at $\left.30^{\circ} \mathrm{C}\right)$, the rate of unstimulated chemiluminescence was approximately 250 c.p.s. per $10^{6}$ cells, but upon the addition of $1 \mathrm{~mm}$ cyanide there was a rapid increase in this photon emission to 750 c.p.s. per $10^{6}$ cells (Fig. 1, A). The rate of chemiluminescence rapidly declined over the next

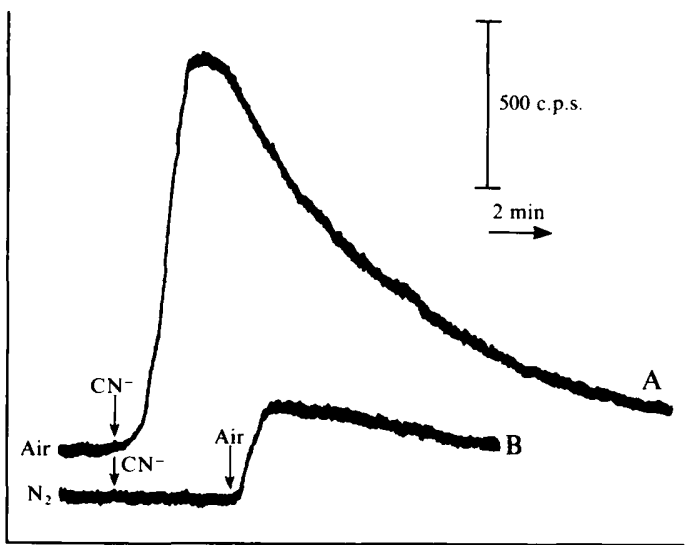

Fig. 1. Lucigenin-dependent chemiluminescence. A. castellanii were grown to the mid-exponential phase $\left(2 \times 10^{6}\right.$ cells $\left.\mathrm{ml}^{-1}\right)$, harvested and

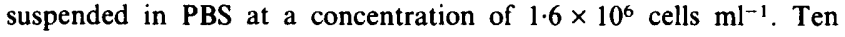
millilitres of this suspension was placed in the 'open' $\mathrm{O}_{2}$ electrode/chemiluminescence system (Edwards et al., 1987), which was maintained at $30^{\circ} \mathrm{C}$, and lucigenin was added to $50 \mu \mathrm{M}$ (final concn). At the point indicated $(\downarrow) 1 \mathrm{~mm}$-sodium cyanide was added. In (A) the gas phase comprised air $\left(235 \mu \mathrm{M}-\mathrm{O}_{2}\right)$; in (B) the gas phase comprised $\mathrm{O}_{2}$-free $\mathrm{N}_{2}$, and at the point indicated $(\downarrow)$ the gas phase was switched to air, using a digital gas mixer.

$15 \mathrm{~min}$, but could be restored if a further addition of cyanide was made (data not shown). This phenomenon is explained by the fact that the inhibitor is expelled from the liquid phase in such 'open' systems because of the continuous gas flow through the suspension (Lloyd et al., $1979 \mathrm{~b}$ ). When suspensions were incubated under anaerobic conditions (by replacing air with $\mathrm{O}_{2}$-free $\mathrm{N}_{2}$ in the gas phase, and monitoring the $\mathrm{O}_{2}$ tension in the liquid) the endogenous rate of chemiluminescence was decreased to 150 c.p.s. per $10^{6}$ cells and no increase in chemiluminescence was observed upon the addition of cyanide (Fig. 1, B). However, when the suspension was aerated by switching the gas phase to air, photon emission was detected. Additions of sodium azide (up to $5 \mathrm{mM}$ ) did not stimulate lucigenin-chemiluminescence (data not shown).

When $10 \mu \mathrm{M}$-luminol replaced lucigenin in the incubations, a similar chemiluminescence emission was observed upon the addition of $1 \mathrm{~mm}$-cyanide (Fig. 2, B), but the responses were lower than those observed for lucigenin (although it must be pointed out that the concentrations of luminol used in these experiments were lower than those of lucigenin). For example, unstimulated levels of photon emission were 200 c.p.s. per $10^{6}$ cells, which increased by a further 125 c.p.s. per $10^{6}$ cells upon the addition of cyanide. A similar $\mathrm{O}_{2}^{-}$dependence of luminol-chemiluminescence was observed (Fig. 2, C) but again, sodium azide did not elicit a response (Fig. 2, A).

No differences in cyanide-stimulated lucigenin- or 


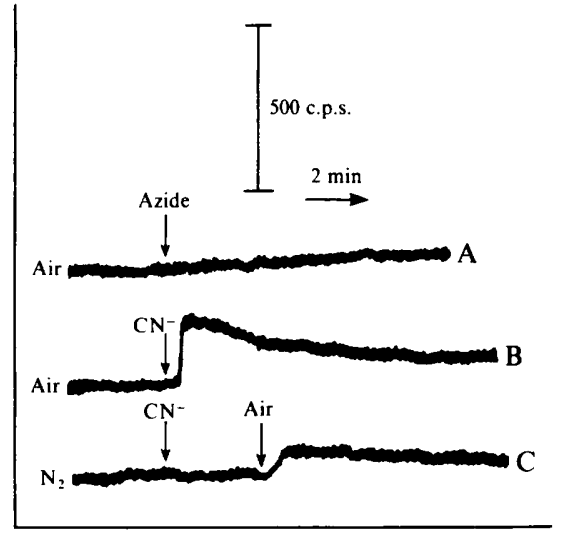

Fig. 2. Luminol-dependent chemiluminescence. Experimental conditions were exactly as described in the legend to Fig. 1 except that the suspensions contained $10 \mu \mathrm{M}$-luminol. In (A) $1 \mathrm{~mm}$-sodium azide was added and in (B) $1 \mathrm{~mm}$-sodium cyanide was added, at the points indicated $(\downarrow)$ : the gas phase comprised air. In (C) the gas phase comprised $\mathrm{O}_{2}$-free $\mathrm{N}_{2}$ and at the point indicated $(\downarrow) 1 \mathrm{mM}$-sodium cyanide was added and then at $(\downarrow)$ the gas phase was switched to air.

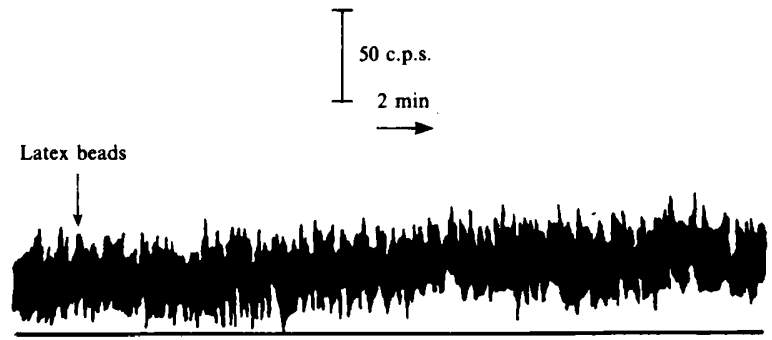

Fig. 3. Lucigenin-dependent chemiluminescence during phagocytosis. Experimental conditions were as described in the legend to Fig. 1, the gas phase comprising $235 \mu \mathrm{M}-\mathrm{O}_{2}$. At the point indicated $(\downarrow)$ latex beads $\left(3 \times 10^{10}, 1 \mu \mathrm{m}\right.$ diameter in a total volume of $\left.10 \mathrm{ml}\right)$ were added and the chemiluminescence response measured.

luminol-chemiluminescence were observed when cells were isolated from cultures which were harvested at the early (cyanide-stimulated) or late (cyanide-sensitive) phases of exponential growth (Edwards \& Lloyd, 1977), indicating no dependency on the activities of the alternative terminal oxidases.

\section{Cyanide-stimulated and phagocytosis-induced chemiluminescence and $\mathrm{O}_{2}^{-}$production}

We have previously shown that phagocytosis of heatkilled yeast or latex beads by $A$. castellanii is accompanied by increased rates of lucigenin-dependent, but not luminol-dependent, chemiluminescence and $\mathrm{O}_{\overline{2}}^{-}$ production (Davies et al., 1991). Therefore, it was necessary to ascertain if cyanide-stimulated chemiluminescence was related to the molecular processes which are responsible for the respiratory burst and which

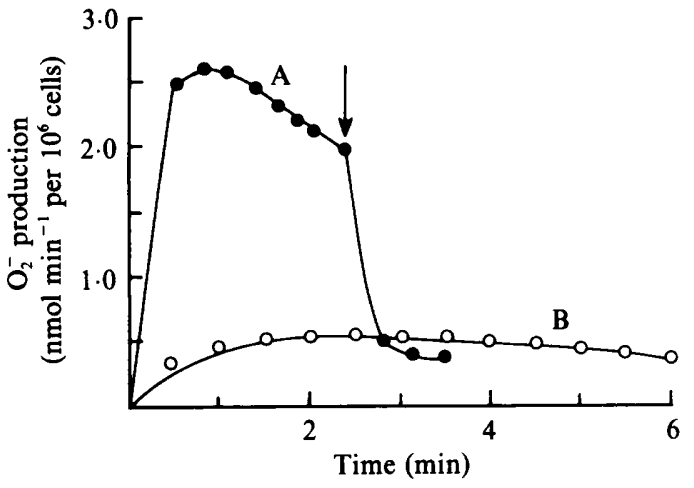

Fig. 4. Cyanide-stimulated $\mathrm{O}_{2}^{-}$production. $A$. castellanii were grown to the mid-exponential phase of growth and suspended in PBS at a concentration of $1 \times 10^{6} \mathrm{cells} \mathrm{ml}^{-1}$. Cytochrome $c$ was added to a final concentration of $75 \mu \mathrm{M}$ and superoxide dismutase $\left(20 \mu \mathrm{g} \mathrm{ml}^{-1}\right)$ was added to the reference cuvette. $A_{550}$ was measured using a PerkinElmer Lambda 5 spectrophotometer. After following endogenous activity for $5 \mathrm{~min}, 1 \mathrm{~mm}$-cyanide was added to both sample and reference cuvettes and the increase in $A_{550}$ measured. In (A) $20 \mu \mathrm{g} \mathrm{ml}^{-1}$ (final concn) superoxide dismutase was added to the sample cuvette at the point indicated $(\downarrow)$, whereas in (B) this was added to the suspension prior to the addition of cyanide.

become activated during phagocytosis. Under identical experimental conditions to those in Figs. 1 and 2, the lucigenin-chemiluminescence activated upon phagocytosis of latex beads by $A$. castellanii was just detectable above background levels (Fig. 3) using this apparatus. In order to measure rates of $\mathrm{O}_{2}^{-}$production, cells were suspended in PBS containing cytochrome $c$, and $\mathrm{O}_{2}^{-}$ generation was measured as the rate of superoxidedismutase-inhibitable reduction of this haemoprotein (Babior et al., 1973). Endogenous rates of cytochrome $c$ reduction were zero, indicating immeasurable rates of $\mathrm{O}_{2}^{-}$production in untreated cells, but upon the addition of 1 mM-cyanide, the rate of reduction increased rapidly, reaching a maximal rate of $2.5 \mathrm{nmol} \mathrm{min}{ }^{-1}$ per $10^{6}$ cells (Fig. 4, A). This cytochrome $c$ reduction was abolished upon addition of superoxide dismutase (at $20 \mu \mathrm{g} \mathrm{ml}^{-1}$ ), indicating its dependence upon the generation of $\mathrm{O}_{2}^{-}$. When suspensions were incubated with superoxide dismutase prior to the addition of cyanide, the rate of cytochrome $c$ reduction was greatly reduced (Fig. 4, B), confirming that the reduction of this haemoprotein was indeed due to the formation of $\mathrm{O}_{2}^{-}$. We have previously shown that upon phagocytosis of latex beads by $A$. castellanii, $\mathrm{O}_{2}^{-}$generation is also rapidly activated and maximal rates of production are $1 \cdot 8-2 \cdot 1 \mathrm{nmol} \mathrm{min}^{-1}$ per $10^{6}$ cells (Davies et al., 1991). Hence, in view of the comparable activated rates of $\mathrm{O}_{2}^{-}$generation but disparate levels of lucigenin-chemiluminescence, we conclude that $\mathrm{O}_{2}^{-}$generation via phagocytosis and upon cyanide addition arise from two separate molecular processes. 


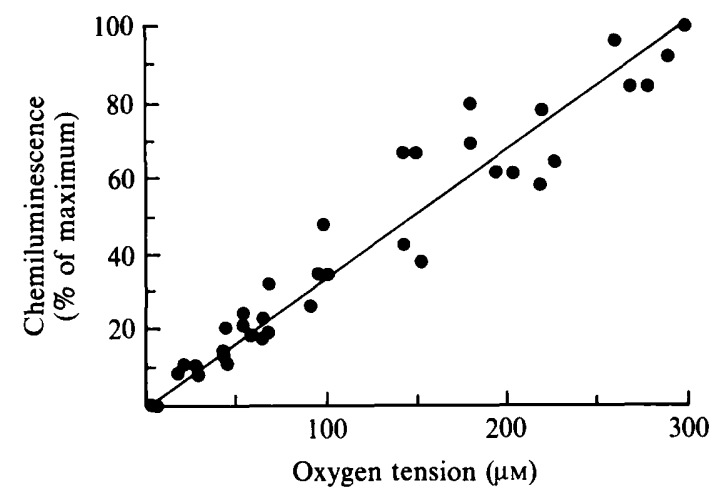

Fig. 5. $\mathrm{O}_{2}$-dependence of cyanide-stimulated chemiluminescence. Cells were incubated as described in the legend to Fig. 1 at $5 \times 10^{5}$ cells ml-1. $^{-1}$. $\mathrm{O}_{2}$ tensions in the suspensions were poised at pre-determined levels using a digital gas mixture and after a 5 min equilibration period, $1 \mathrm{~mm}$ cyanide was added and the chemiluminescence response measured. $0 \%$ indicates the unstimulated background level and $100 \%$ represents 1000 c.p.s. per $10^{6}$ cells.

\section{$\mathrm{O}_{2}^{-}$dependence of cyanide-stimulated chemiluminescence}

In order to determine the relative $\mathrm{O}_{2}$-dependence of cyanide-stimulated chemiluminescence in $A$. castellanii, cells were suspended in PBS (containing $50 \mu \mathrm{M}$-lucigenin) and the $\mathrm{O}_{2}$ tension in the medium (monitored using an $\mathrm{O}_{2}$ electrode) was poised at pre-determined levels throughout the range $0-235 \mu \mathrm{M}$. After equilibration at each $\mathrm{O}_{2}$ tension for $5 \mathrm{~min}, 1 \mathrm{mM}$-cyanide was added to the suspension through an injection port so that the $\mathrm{O}_{2}$ tension was not appreciably perturbed, and the magnitude of the chemiluminescence response was measured. Fig. 5 shows that the rate of cyanidestimulated chemiluminescence was directly proportional to the $\mathrm{O}_{2}$ tension in the medium. When suspensions were gassed with $\mathrm{O}_{2} / \mathrm{N}_{2}$ mixtures to attain $\mathrm{O}_{2}$ tensions in the medium above $235 \mu \mathrm{M}$ (the $\mathrm{O}_{2}$ tension of air-saturated medium at $30{ }^{\circ} \mathrm{C}$ ), the rate of cyanide-stimulated chemiluminescence increased further. Thus, at $300 \mu \mathrm{M}$ $\mathrm{O}_{2}$, the activated response was approximately $20 \%$ greater than that observed at $235 \mu \mathrm{M}-\mathrm{O}_{2}$. These data indicate that under hyperoxic conditions, when it may be predicted that oxidant-scavenging systems such as superoxide dismutase activity may be saturated due to oxidative stress, rates of oxidant production detected by lucigenin-chemiluminescence are enhanced.

\section{Effect of diethyldithiocarbamate on chemiluminescence}

The above experiments confirm the idea that cyanide stimulates $\mathrm{O}_{2}^{-}$production in $A$. castellanii via inhibition of superoxide dismutase (Lloyd et al., 1979b). However, cyanide also inhibits a number of other enzymes involved in oxidative metabolism in these cells (such as

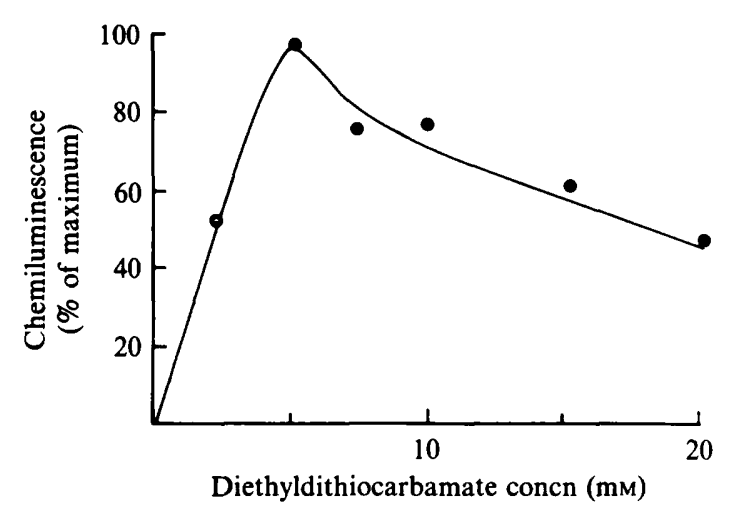

Fig. 6. Effect of diethyldithiocarbamate concentration on lucigenindependent chemiluminescence. Experimental conditions were as described in the legend to Fig. 1 except that the cell concentration was $5 \times 10^{5}$ cells ml$^{-1}$. Rates of endogenous lucigenin-dependent chemiluminescence were measured and then diethyldithiocarbamate was added at the final concentration indicated. The rate of chemiluminescence was monitored and the maximal rate obtained (by $30 \mathrm{~min}$ ) was plotted as a function of the inhibitor concentration: $100 \%$ (the rate obtained at a concentration of $5 \mathrm{mM}$ ) represents 250 c.p.s. per $10^{6}$ cells, whilst $0 \%$ represents the unstimulated background level.

cytochrome oxidase and catalase) and hence its effects on lucigenin-dependent chemiluminescence may not be solely due to inhibition of superoxide dismutase activity. Therefore, a more specific inhibitor of superoxide dismutase was sought, and to this end, the effects of diethyldithiocarbamate (Halliwell \& Gutteridge, 1985) on this phenomenon were tested.

Suspensions of $\boldsymbol{A}$. castellanii were incubated with various concentrations of diethyldithiocarbamate and subsequent rates of lucigenin-dependent chemiluminescence were measured. This inhibitor did indeed stimulate chemiluminescence, but its effects were different to those of cyanide in a number of ways. Firstly, the inhibitor induced a much more gradual increase in chemiluminescence compared with cyanide, being maximal by 30 min after addition: this may reflect a reduced ability of diethyldithiocarbamate to permeate the cells. Secondly, the maximal rate of chemiluminescence stimulated by diethyldithiocarbamate was only about one-third of that stimulated by cyanide. Thirdly, the diethyldithiocarbamate-stimulated chemiluminescence signal was more stable than that induced by cyanide, presumably because the former inhibitor was not expelled from the suspension during continuous aeration. The maximal enhancement of lucigenin-dependent chemiluminescence by diethyldithiocarbamate occurred at a concentration of $5 \mathrm{~mm}$ (Fig. 6) and this concentration of inhibitor was used in experiments to determine the $\mathrm{O}_{2}$-dependence of its action.

Suspensions of $A$. castellanii were incubated with $50 \mu \mathrm{M}$-lucigenin at an $\mathrm{O}_{2}$ tension of $300 \mu \mathrm{M}$ and diethyl- 


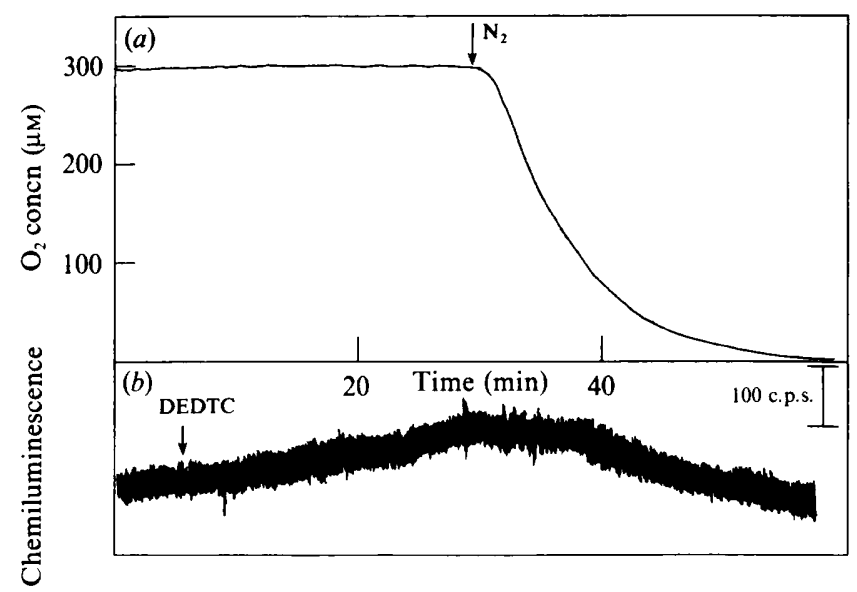

Fig. 7. Effect of $\mathrm{O}_{2}$ on diethyldithiocarbamate-stimulated lucigeninchemiluminescence. Experimental conditions were as described in the legend to Fig. 1 except that the cell concentration was $5 \times 10^{5}$ cells $\mathrm{ml}^{-1}$. The cells were incubated at an $\mathrm{O}_{2}$ tension of $300 \mu \mathrm{M}$ and at the point indicated $(\downarrow)$ diethyldithiocarbamate (DEDTC) was added to a final concentration of $5 \mathrm{~mm}$. The chemiluminescence response was then measured and the gas phase was then switched to $\mathrm{N}_{2},(\downarrow)(a)$ shows the $\mathrm{O}_{2}$ tension in the suspension whilst $(b)$ shows the lucigenindependent chemiluminescence.

dithiocarbamate was added at a concentration of $5 \mathrm{~mm}$ (Fig. 7). The rate of chemiluminescence then increased slowly to reach a maximal value after about $30 \mathrm{~min}$ incubation. This rate was stable for up to a further $2 \mathrm{~h}$. The suspension was then made anaerobic by switching the gas phase to $\mathrm{N}_{2}$ and the rate of chemiluminescence declined as the $\mathrm{O}_{2}$ tension in the suspension decreased, so that at zero $\mathrm{O}_{2}$, the rate of chemiluminescence was equivalent to that of the unstimulated suspension. This approach was then modified to measure the $\mathrm{O}_{2}$ dependence of diethyldithiocarbamate-stimulated lucigenin-dependent chemiluminescence. Suspensions of cells were incubated at an $\mathrm{O}_{2}$ tension of $300 \mu \mathrm{M}$ and diethyldithiocarbamate added to $5 \mathrm{mM}$. After $30 \mathrm{~min}$ incubation (when the rate of chemiluminescence had reached a steady value) the suspension was made anaerobic by switching the gas phase to $\mathrm{N}_{2}$. As the $\mathrm{O}_{2}$ tension decreased to $140 \mu \mathrm{M}$, there was only about a $15 \%$ reduction in the chemiluminescence emission, but below this tension, the rate of photon emission was more $\mathrm{O}_{2}$ dependent (Fig. 8). At zero $\mathrm{O}_{2}$, the rate of chemiluminescence was identical to that of unstimulated suspensions. However, when anaerobic suspensions were then re-aerated, the $\mathrm{O}_{2}$ profile observed was quite different to that of aerobic suspensions becoming anaerobic. For example, at $\mathrm{O}_{2}$ tensions up to $140 \mu \mathrm{M}$, the rate of chemiluminescence was always lower in anaerobic $\rightarrow$ aerobic suspensions than in aerobic $\rightarrow$ anaerobic suspensions. Above $140 \mu \mathrm{M}-\mathrm{O}_{2}$, the anaerobic $\rightarrow$ aerobic

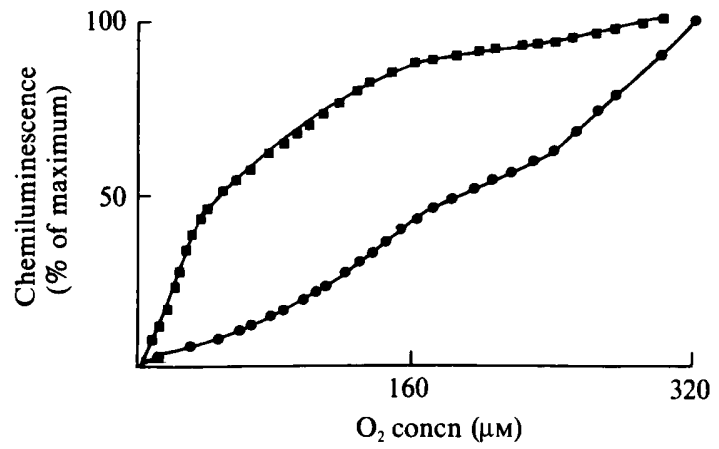

Fig. 8. $\mathrm{O}_{2}$ dependence of diethyldithiocarbamate-stimulated lucigeninchemiluminescence. Experimental conditions were as described in the legend to Fig. 1 except that the cell concentration was $5 \times 10^{5}$ cells $\mathrm{ml}^{-1}$. Cells were incubated at $300 \mu \mathrm{M}-\mathrm{O}_{2},(\square)$ and diethyldithiocarbamate was added to a final concentration of $5 \mathrm{~mm}$. After a stable chemiluminescence response was obtained $(30 \mathrm{~min})$ the gas phase was switched to $\mathrm{N}_{2}$ and the rate of chemiluminescence was measured as a function of the $\mathrm{O}_{2}$ tension. Anaerobic suspensions (O) were then reaerated by switching the gas phase to $300 \mu \mathrm{M}-\mathrm{O}_{2}$. Similar results were obtained in five other experiments (data not shown).

chemiluminescence was much more $\mathrm{O}_{2}$ dependent and when tensions approached $300 \mu \mathrm{M}$, maximal rates of chemiluminescence were restored. Several identical cycles of chemiluminescence changes during aerobic $\rightarrow$ anaerobic $\rightarrow$ aerobic transitions could be monitored. These data indicate that anaerobic suspensions may be adapting to increasing $\mathrm{O}_{2}$ tensions in the environment by reducing the activities of $\mathrm{O}_{2}^{-}$-generating pathways which may be potentially hazardous.

\section{Discussion}

The data presented here confirm and extend the idea that the respiratory inhibitor sodium cyanide induces chemiluminescence in the amoeba $A$. castellanii (Lloyd et al., $1979 b$ ) and strongly indicate that this phenomenon is due, at least in part, to inhibition of superoxide dismutase which leads to the accumulation of $\mathrm{O}_{2}^{-}$: this radical itself, or species formed from it (such as lipid peroxides, carbonyl radicals, etc.), may be the oxidant species which are responsible for the photon emission detected by the probes used in this study (luminol or lucigenin). Because no differences in cyanide-stimulated chemiluminescence were detected in cultures isolated from either the early (cyanide-stimulated) or late (cyanide-sensitive) exponential phases of growth (Edwards \& Lloyd, 1977), we conclude that the phenomenon of cyanide-stimulated photon emission is independent of the activities of the phosphorylating or alternative mitochondrial oxidases known to operate in these organisms. Mitochondrial oxidase involvement may also 
be ruled out on the basis of the $\mathrm{O}_{2}$-dependence of the phenomenon (Lloyd et al., 1983a).

Previous work has shown that like 'professional phagocytes' such as neutrophils and macrophages, $A$. castellanii can generate $\mathrm{O}_{2}^{-}$during a cyanide-insensitive respiratory burst of $\mathrm{O}_{2}$ uptake which is activated upon phagocytosis of latex beads or heat-killed yeast (Davies et al., 1991). Furthermore, this process results in the generation of lucigenin-dependent, but not luminoldependent, chemiluminescence, although the levels of photon emission (on a per cell basis) are some 200 -fold lower than those observed in neutrophils. Luminolchemiluminescence can be detected, however, when exogenous myeloperoxidase is added to phagocytosing suspensions of $A$. castellanii and this observation is explained by the fact that, unlike lucigenin, luminol requires the activity of an $\mathrm{O}_{2}^{-} / \mathrm{H}_{2} \mathrm{O}_{2}$-generating system and a peroxidase for maximal efficiency of photon emission. A. castellanii possesses no endogenous peroxidase activity and hence lucigenin-chemiluminescence is a more efficient probe for $\mathrm{O}_{2}^{-} / \mathrm{H}_{2} \mathrm{O}_{2}$ than luminol in this experimental system.

Upon the addition of cyanide, but not azide, both lucigenin- and luminol-chemiluminescence were stimulated in suspensions of $A$. castellanii although the rate of photon emission in the presence of the former probe was about fivefold greater than that detected with the latter probe. Furthermore, cyanide, but not azide, stimulated superoxide-dismutase-inhibitable cytochrome $c$ reduction and hence this assay provides direct evidence for the increased generation of $\mathrm{O}_{2}^{-}$upon addition of this inhibitor. Similarly, phagocytosis of latex beads induces lucigenin-chemiluminescence, but this was barely detectable above background photon emission. In contrast, rates of cyanide-stimulated and phagocytosis-induced $\mathrm{O}_{2}^{-}$production were comparable. Hence, we conclude that the molecular pathways which result in cyanidestimulated and phagocytosis-induced $\mathrm{O}_{2}^{-}$production, and lucigenin-chemiluminescence, are distinct from one another.

One likely process that may produce chemiluminescence upon addition of cyanide is that proposed by Lloyd et al. (1979b), namely inhibition of superoxide dismutase and subsequent accumulation of $\mathrm{O}_{2}^{-}$. However, cyanide is a non-specific inhibitor of a number of haemoproteins involved in oxidative metabolism (such as cytochrome oxidase and catalase) and hence we sought a more specific inhibitor of superoxide dismutase. Therefore, we measured the effect of diethyldithiocarbamate on lucigenin-chemiluminescence and showed that this also could activate oxidant production. Its major difference to the action of cyanide was that it took longer to exert its effects (presumably because of permeability problems) but the chemiluminescence signal obtained was more stable. This allowed us to determine the relative $\mathrm{O}_{2}$ affinities of diethyldithiocarbamate-stimulated $\mathrm{O}_{2}^{-}$production and also to examine the effects of aerobic/anaerobic transitions on this phenomenon.

When the $\mathrm{O}_{2}$-dependency of cyanide-stimulated chemiluminescence was measured by poising the $\mathrm{O}_{2}$ tensions in the suspensions prior to addition of inhibitor, there was a close correlation between the chemiluminescence response obtained and the $\mathrm{O}_{2}$ tension between 0 and $300 \mu \mathrm{M}$. This observation fits the idea that rates of $\mathrm{O}_{2}^{-}$production are directly proportional to the $\mathrm{O}_{2}$ tension in the environment and that conditions of oxidative stress are associated with high levels of production of this oxidant: steady-state levels of $\mathrm{O}_{2}^{-}$are thus normally regulated by superoxide dismutases which prevent the accumulation of hazardous levels of this species. Whilst $\mathrm{O}_{2}^{-}$itself has only limited reactivity, other radical species invariably generated from this, such as $\mathrm{OH}$, are extremely damaging. Similarly, diethyldithiocarbamatestimulated chemiluminescence showed $\mathrm{O}_{2}$-dependence from 0 to $300 \mu \mathrm{M}-\mathrm{O}_{2}$, but in these experiments an unusual and striking adaptation to anaerobiosis was observed: when anaerobic suspensions were re-aerated, rates of lucigenin-chemiluminescence were much lower than those observed in corresponding aerobic cultures which had been made anaerobic. This may be interpreted as an attempt by the cells to shut down oxidative processes leading to $\mathrm{O}_{2}^{-}$production in order to prevent the likelihood of tissue damage mediated either directly or indirectly by this oxidant. Adaptation of mitochondrial respiration to periods of anaerobiosis has previously been reported in this organism (Lloyd et al., 1983b)

In conclusion, we have shown that $A$. castellanii possesses at least two distinct oxidative pathways that result in $\mathrm{O}_{2}^{-}$formation and lucigenin-chemiluminescence. The first of these occurs via the respiratory-burst enzyme which is activated upon phagocytosis: this enzyme appears analogous to the NADPH oxidase of 'professional phagocytes' and may be instrumental in microbial killing. The second source of $\mathrm{O}_{2}^{-}$arises from the inhibition of superoxide dismutase by cyanide or diethyldithiocarbamate. Further work is necessary to determine if perturbation of the activity of this enzyme is associated with features of oxidative stress, such as increased rates of lipid peroxidation.

\section{References}

ALLEN, R. C. (1981). Lucigenin chemiluminescence: a new approach to the study of polymorphonuclear leukocyte redox activity. In Bioluminescence and Chemiluminescence, pp. 63-73. Edited by M. A. De Luca. New York: Academic Press.

AlleN, R. C. \& Loose, L. D. (1976). Phagocytic activation of a luminol-dependent chemiluminescence in rabbit alveolar and peritoneal macrophages. Biochimica et Biophysica Acta 96, 245-252. 
Babior, B. M., Kipnes, R. S. \& CurnutTe, J. T. (1973). Biologica defense mechanisms: the production by leukocytes of superoxide, a potential bactericidal agent. Journal of Clinical Investigation 52, 741744.

CURNutTe, J. T. (editor) (1988). Phagocyte Defects I and II. Hematology/Oncology Clinics of North America. Philadelphia, USA: Saunders.

Davies, B., Chattings, L. S. \& Edwards, S. W. (1991). Superoxide generation during phagocytosis by Acanthamoeba castellanii: similarities to the respiratory burst of immune phagocytes. Journal of General Microbiology 137, 705-710.

EDWARDS, S. W. (1987). Luminol- and lucigenin-dependent chemiluminescence of neutrophils: role of degranulation. Journal of Clinical and Laboratory Immunology 22, 35-39.

EdWARDS, S. W. \& LLOYD, D. (1977). Cyanide-insensitive respiration in Acanthamoeba castellanii. Changes in sensitivity of whole cell respiration during exponential growth. Journal of General Microbiology 103, 207-213.

Edwards, S. W. \& Lloyd, D. (1978). Properties of mitochondria isolated from cyanide-sensitive and cyanide-stimulated cultures of Acanthamoeba castellanii. Biochemical Journal 174, 203-211.
EDWARDS, S. W., SAY, J. E. \& HarT, C. A. (1987). Oxygen-dependent killing of Staphylococcus aureus by human neutrophils. Journal of General Microbiology 133, 3591-3597.

Halliwell, B. \& GuTteridge, J. M. C. (1985). Free Radicals in Biology and Medicine. Oxford: Clarendon Press.

Lloyd, D., EdWards, S. W., Kristensen, B. \& Degn, H. (1979a). The effects of inhibitors on the oxygen kinetics of terminal oxidases of Acanthamoeba castellanii. Biochemical Journal 182, 11-15.

Lloyd, D., Boveris, A., Reiter, R., Filipowski, M. \& Chance, B. $(1979 b)$. Chemiluminescence in Acanthamoeba castellanii. Biochemical Journal 184, 149-156.

LLOYD, D., Mellor, H. \& Williams, J. L. (1983a). Oxygen affinity of the respiratory chain of Acanthamoeba castellanii. Biochemical Journal 214, 47-51.

Lloyd, D., Protheroe, R., Williams, T. N. \& Williams, J. L. $(1983 \mathrm{~b})$. Adaptation of the respiratory system of Acanthamoeba castellanii to anaerobiosis. FEMS Microbiology Letters 17, 143-146.

SEGAL, A. W. \& Jones, O. T. G. (1978). Novel cytochrome $b$ system in phagocytic vesicles of human granulocytes. Nature, London 276, 515517. 